\title{
Is p53 Overexpression a Predictor of Worse Outcomes in Endometrioid Endometrial Carcinomas?
}

\author{
Raid Jastania ${ }^{1}$ and Tahani Nageeti ${ }^{*}$ \\ ${ }^{1}$ Assistant Professor, Faculty of Medicine, Umm Al-Qura University, Saudi Arabia \\ ${ }^{2}$ Consultant Radiation Oncology, Radiation Oncology Department, Jeddah Oncology Centre, King Abdullah \\ Medical City Holy Capital Saudi Arabia
}

*Corresponding author: Tahani Nageeti, MBChB, FRCPC, Consultant Radiation Oncology, Radiation Oncology Department, Jeddah Oncology Centre, King Abdulla Medical City, Holly Capital, PO Box: 7700, Makkah 24246, Saudi Arabia, Tel: 012-56650000

\begin{abstract}
Endometrial cancer (EC) is the most common neoplasm of the female genital tract. Although immunohistochemical methods have been used for detecting several biomarkers for several cancer types, this method is still underutilized in EC. We conducted this review to identify the currently available published evidence to support the utilization of immunohistochemical for p53 protein overexpression as prognostic and predictive biomarkers of disease outcomes in endometrioid endometrial carcinoma.
\end{abstract}

\section{Keywords}

p53, Endometrioid endometrial carcinomas, Outcomes

\section{Introduction}

Endometrial cancer (EC) is the most common neoplasm of the female genital tract and the fourth most common cancer occurring in women after breast, lung and bowel cancers [1]. The age-standardized incidence for a diagnosis of endometrial cancer is 13.6 per 100,000 women and accumulative risk of $1.71 \%$ [2]. It is derived from the endometrial epithelial lining of the uterine corpus. The Surveillance, epidemiology and end results (SEER) data from 2003-2009 showed almost $(70 \%)$ of EC cases were diagnosed at an early stage, and about $(30 \%)$ were diagnosed with regional or distant metastasis [3]. It has been observed that there is an increase in mortality related to endometrial cancers [4].

There are two main types have been recognized. The most common type is type-I referred to endometrioid adenocarcinoma, which represents more than $80 \%$ of endometrial carcinomas. These tumors are related to estrogen stimulation and can develop in peri- or postmenopausal women. They are predominantly low-grade endometrioid adenocarcinomas, confined to the uterus and often are preceded by endometrial hyperplasia. TypeII tumors are non-endometrioid high-grade carcinomas which often invade deeply into the myometrium and follow an aggressive clinical behavior. These tumors are not related to estrogen stimulation and often occur in older women [5].

Endometrial adenocarcinoma cancer is more of heterogeneous tumors that each group should be dealt with differently. In clinical practice, most often postsurgery single or multimodalities adjuvant therapy is recommended based on clinicopathological risk factors that predict for worsening local or systemic relapse and survival. These prognostic factors including age, race, histologic subtype, surgical stage, histological grade and presence of lymphovascular space invasion (LVI).

Although immunohistochemical methods have been used for detecting several biomarkers of possible prognostic importance for several malignancies, this method is underutilized as prognostic tools in endometrial neoplasms.

To improve overall disease outcomes in endometrioid endometrial carcinomas, we need to identify the group of patients with tumors that carry poor prognostic behaviors and treat them appropriately with adjuvant and possibly targeted therapy.

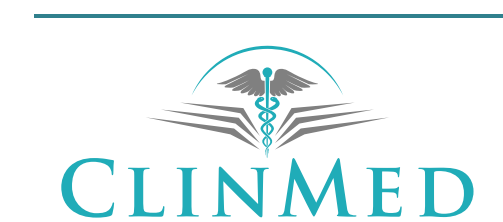

INTERNATIONAL LIBRARY

Citation: Jastania R, Nageeti T (2018) Is p53 Overexpression a Predictor of Worse Outcomes in Endometrioid Endometrial Carcinomas?. Int J Pathol Clin Res 4:081. doi.org/10.23937/2469-5807/1510081 Accepted: November 20, 2018: Published: November 22, 2018

Copyright: (c) 2018 Jastania R, et al. This is an open-access article distributed under the terms of the Creative Commons Attribution License, which permits unrestricted use, distribution, and reproduction in any medium, provided the original author and source are credited. 
Table 1: Summary of studies comparing p53 positive group vs. p53 negative group in endometrial carcinomas.

\begin{tabular}{|c|c|c|c|}
\hline Study & Number of Patients & Histology & Outcomes \\
\hline Fadare, et al. [7] & 50 & Clear cell carcinomas & $\begin{array}{ll}\text { - } & \text { Recurrence } 73 \% \text { in p53 positive } \\
& \text { group }(P<0.008) \\
\text { - } & \text { PFS } 56 \text { vs. } 88 \text { months }(P<0.01) \\
\text { - } & \text { OS } 63 \text { vs. } 83 \text { months }(P=0.07)\end{array}$ \\
\hline Garg, et al. [9] & 35 & Endometrioid endometrial carcinomas & $\begin{array}{l}\text { - } 3 \text {-year PFS } 52 \% \text { vs. } 94 \%(P=0.02) \\
\text { - } 3 \text {-year DSS } 54 \% \text { vs. } 100 \%(P< \\
\text { 0.003) }\end{array}$ \\
\hline Catasus, et al. [10] & 132 & $\begin{array}{l}\text { Endometrial carcinomas } \\
\begin{aligned}- & (77 \%) \text { endometrioid } \\
& \text { adenocarcinomas } \\
- & (11 \%) \text { non-endometrioid } \\
& \text { adenocarcinomas } \\
- & (12 \%) \text { mixed endometrioid } \\
& \text { adenocarcinomas-non- } \\
& \text { endometrioid adenocarcinomas }\end{aligned}\end{array}$ & $\begin{array}{l}\text { - Kaplan-Meier Survival Analysis ( } P= \\
0.000)\end{array}$ \\
\hline Huvila, et al. [11] & 306 & Endometrioid endometrial carcinomas & $\begin{array}{l}\text { A } 30 \text {-fold risk of dying of disease in } \\
\text { high-risk group compared to low-risk } \\
\text { group }\end{array}$ \\
\hline Obata, et al. [12] & 154 & Endometrioid endometrial carcinomas & $\begin{array}{ll}\text { - } & \text { Recurrence or Metastasis } \\
\text { - } & \text { Grade } 1-2: 37.8 \% \text { vs. } 5.3 \%(P< \\
& 0.01) \\
\text { - } & \text { PFS } 61.1 \% \text { vs. } 21.9 \%(P<0.01) .\end{array}$ \\
\hline
\end{tabular}

PFS: Progression Free Survival; OS: Overall Survival; DSS: Disease-Specific Survival.

We conducted this review to identify the currently available published evidence to support the adding value of utilization of immunohistochemical test for $\mathrm{p} 53$ protein overexpression as prognostic and predictive biomarkers of disease outcomes in endometrioid endometrial carcinoma.

\section{Discussion}

There are growing evidence to support the prognostic value of overexpression of $\mathrm{p} 53$ in endometrial carcinomas (Table 1). Although p53 alteration present in almost $90 \%$ of non-endometrioid endometrial carcinomas, they are still present in $10-20 \%$ of endometrioid endometrial carcinoma [6]. In non-endometrioid endometrial carcinomas, p53 protein overexpression was significantly associated with lower overall and progression-free survival. In a study of 50 endometrial clear cell carcinomas, (34\%) were p53 positive, and (66\%) of cases had a p53 wild-type (p53-wt) immunophenotype, $73 \%$ of recurrences were in the p53 positive group $(P<0.008)$. On univariate analyses, the median overall survival was significantly lower for p53 positive vs. p53-wt cases (63 vs. 83 months) $(P<0.07)$, and the median progression-free survival was significantly lower (56 vs. 88 months) $(\mathrm{P}<0.01)$. On multivariate analyses, p53 expression was not associated with reduced overall or progression-free survival or pathologic stage or morphologic patterns [7]. A case report of endometrioid endometrial carcinoma with spindle cell differentiation showed overexpression of p16 and p53 indicating that there is a spectrum of these tumors [8]. In most clinical practice, the prognosis of endometrioid endometrial carcinomas still relies on conventional clinicopathological prognostic factors. We found three published clinical data on utilizing p53 immunohistochemistry as an additional tool to predict disease outcomes in endometrioid endometrial carcinomas.

A study was published on the application of p53 immunohistochemistry as an objective prognostic marker in 35 cases of a subset of morphologically ambiguous endometrial carcinomas. Overexpression of p53 defined as (IHC score $\geq 9$ ) was seen in 17 of 35 cases. They found tumors with p53 overexpression were associated with inferior clinical outcomes including, progression-free survival and disease-specific survival compared with those without overexpression. The 3 -year progression-free survival was $94 \%$ vs. $52 \%$, and disease-specific survival was $100 \%$ vs. $54 \%$ in patients with no $\mathrm{p} 53$ overexpression vs. patients with p53 overexpression ( $P=0.02$ and 0.003 , respectively). Additionally, a histologic feature that correlated most with p53 overexpression was the presence of diffuse high nuclear grade [9]. Another comparative clinicopathologic and molecular genetic study of 132 endometrial carcinomas found p53 alterations (strong expression or mutations), significantly, more present in non-endometrioid adenocarcinomas (54\%) and mixed endometrioid adenocarcinomas-non-endometrioid adenocarcinomas (50\%) than in low-grade endometrioid adenocarcinomas (2\%) or in high-grade endometrioid adenocarcinomas (17\%). In addition, they found tumors with p53 alterations associated with lymphovascular invasion more frequently (38\%) than tumors without p53 alterations (18.5\%). In this study, patients who had tumors with p53 alterations had a significantly shorter survival than those without them [10]. A recent study on 306 endometrioid endometrial cancer cases, a panel of immunohistochemistry 
including (ER, PR, HER2, Ki-67, MLH1 and p53, L1CAM and ASRGL1) was carried out. P53 and ASRGL1 immunohistochemistry were identified as the most accurate predictor of relapse-free and disease-specific survival. The patients' cohort was classified into high, intermediate and low-risk groups by utilizing this panel. The high-risk patients had a 30 -fold risk of dying of disease compared to the low-risk group [11].

A study of 154 patients with endometrial endometrioid carcinoma, suggested that increases in ER $\beta$ and p53 immunoreactivity were significantly correlated with the incidence of metastasis and recurrence. On a multivariate analysis they found that histological grades, immunohistochemistry of p53 overexpression (> 10\%), and high expression of ER $\beta$ (high-ERB) were independently associated with metastasis or recurrence. In the group with high-ER $\beta$, the rates of metastasis or recurrence were $61.1 \%$ in the p53-stained group vs. $21.9 \%$ in the p53negative group. In the group showing deep myometrial invasion showing, (80\%) were positive for both p53stained and high-ER $\beta$. The disease-free survival of patients who were both positive was significantly lower than that in other patients [12].

\section{Conclusions}

Despite the limited data, we can conclude that utilization of the immunohistochemistry including p53 is useful tool for the differentiation of ambiguous histology of endometrial carcinomas. There is some growing evidence to support that p53 overexpression in endometrioid endometrial carcinomas is associated with worse prognosis and outcomes. Thus, p53 immunohistochemistry assays might be a useful tool for further prognostic stratification of endometrioid endometrial carcinomas worth further studies in prospective settings.

\section{References}

1. Siegel RL, Miller KD, Jemal A (2018) Cancer statistics,
2018. CA Cancer J Clin 68: 7-30.

2. http://globocan.iarc.fr/Pages/fact_sheets_population.aspx

3. Ferlay J, Shin HR, Bray F, Forman D, Mathers C, et al. (2010) Estimates of worldwide burden of cancer in 2008: GLOBOCAN 2008. Int J Cancer 127: 2893-2917.

4. Howlader N, Noone AM, Krapcho M, Garshell J, Neyman $\mathrm{N}$, et al. (2013) SEER cancer statistics review, 1975-2010. National Cancer Institute.

5. (2005) ACOG practice bulletin, clinical management guidelines for obstetrician-gynecologists, number 65, August 2005: Management of endometrial cancer. Obstet Gynecol 106: 413-425.

6. Lax SF, Kendall B, Tashiro H, Slebos RJC, Ellenson LH (2000) The frequency of p53, k-ras mutations, and microsatellite instability differs in uterine endometrioid and serous carcinoma. Cancer 88: 814-824.

7. Fadare O, Gwin K, Desouki MM, Crispens MA, Jones HW, et al. (2013) The clinicopathologic significance of p53 and BAF-250a (ARID1A) expression in clear cell carcinoma of the endometrium. Mod Pathol 26: 1101-1110.

8. Sonawane S, Elfituri O, Zhang Y, Yordan E, Ree N (2018) Endometrioid Endometrial Carcinoma with Spindle CellsAberrant P16 and P53 Expression. Int J Surg Pathol.

9. Garg K, Leitao MM, Wynveen CA, Sica GL, Shia J, et al. (2009) p53 overexpression in morphologically ambiguous endometrial carcinomas correlates with adverse clinical outcomes. Mod Pathol 23: 80-92.

10. Catasus L, Gallardo A, Cuatrecasas M, Prat J (2009) Concomitant PI3K-AKT and p53 alterations in endometrial carcinomas are associated with poor prognosis. Mod Pathol 22: $522-529$.

11. Huvila J, Laajala TD, Edqvist PH, Mardinoglu A, Talve L, et al. (2018) Combined ASRGL1 and p53 immunohistochemistry as an independent predictor of survival in endometrioid endometrial carcinoma. Gynecol Oncol149: 173-180.

12. Obata $T$, Nakamura $M$, Mizumoto $Y$, lizuka $T$, Ono $M$, et al. (2017) Dual expression of immunoreactive estrogen receptor $\beta$ and p53 is a potential predictor of regional lymph node metastasis and postoperative recurrence in endometrial endometrioid carcinoma. PLoS ONE 12. 